\title{
La inconmensurabilidad empírica entre la teoría de la selección natural darwiniana y el diseño inteligente de la teología natural ${ }^{*}$ \\ (The Empirical Incommensurability between the Darwinian Theory of Natural Selection and the Inteligent Design of Natural Theology) \\ Santiago GINNOBILI
}

Received: 25.06.2013

Final version: 16.09 .2013

BIBLID [0495-4548 (2014) 29: 81; pp. 375-394]

DOI: 10.1387 / theoria. 9943

RESUMEN: Suele señalarse la fuerte influencia que la teología natural tuvo sobre Darwin en su conceptualización del fenómeno de la adaptación. La teoría de la selección natural explicaría el mismo fenómeno que los teólogos naturales querían explicar: la adaptación. Recientemente ha sido señalado, sin embargo, que la forma darwiniana de conceptualizar la adaptación es novedosa. Las adaptaciones de la teología natural presupondrían la idea de que los organismos existen para la realización y manutención del equilibrio natural establecido por el creador. El punto de este trabajo discutir esta cuestión, asumiendo el marco del estructuralismo metateórico.

Palabras clave: Darwin; Paley; teología natural; selección natural; inconmensurabilidad empírica; Kuhn; estructuralismo metateórico.

ABSTRACT: The strong influence that natural theology had over Darwin's conceptualization of the adaptation phenomenon is frequently noted. The natural selection theory would explain the same phenomenon that the natural theologist intended to explain: the adaptation. Recently has been claimed, on the contrary, that the Darwinian way of conceptualizing adaptation is new. The concept of adaptation from natural theology presupposes the idea that organisms exist for realization and support of the natural equilibrium stated by the creator. In this work I will discuss this point, assuming the metatheoretical structuralism frame.

Keywords: Darwin; Paley; natural theology; natural selection; empirical incommensurability; Kuhn; metatheoretical structuralism.

\section{Introducción}

No hay tema más interesante y central en la filosofía que el establecimiento del modo en que puede llevarse adelante una discusión racional entre sujetos que difieren en sus estándares evaluativos y en los marcos conceptuales con los que piensan el mundo. Hanson había señalado que existía un sentido interesante en el que Tycho Brahe y Kepler podían no ver lo mismo al dirigir su mirada al mismo atardecer (Hanson 1958, 5). Las figuras de perspectivas reversibles de la Gestalt le permitían dar plausibilidad a este punto, que ha sido relacionado habitualmente con la carga teórica de la observación. Al avanzar en la lectura, Hanson parece estar también interesado en señalar cómo la discusión entre personas que comparten la misma categorización

\footnotetext{
* Este trabajo fue realizado con la ayuda de los proyectos de investigación PICT-2007-1558 (ANPCyT, Argentina) y PIP no 112-201101-01135 (CONICET, Argentina). Agradezco los valiosos comentarios realizados por Daniel Blanco, Gustavo Caponi, Luciano Piazza y Andrea Melamed a versiones previas del trabajo.
} 
del mundo es diferente a la discusión entre personas que no la comparten (Hanson 1958, 87). Este es el motivo central de la obra cumbre de Kuhn, La estructura de las revoluciones científicas (Kuhn 1970). La noción con la que Kuhn se refiere metafóricamente a esta cuestión es la de 'inconmensurabilidad'. ${ }^{1}$ Muchísimo ha sido escrito por críticos y simpatizantes del enfoque kuhniano, y por el mismo Kuhn, al respecto de dicho concepto. Para muchos, la idea de que teorías sucesivas sean inconmensurables pone en jaque la racionalidad y el progreso científico. Para otros, tener en cuenta tales situaciones resulta necesario para establecer los límites y las reglas de la discusión racional. Independientemente de estas discusiones de carácter epistemológico, considero que indudablemente el concepto de inconmensurabilidad (así como el marco conceptual completo ofrecido por Kuhn) resulta fructífero para pensar el cambio científico, y en especial, para pensar los períodos revolucionarios. Incluso para discutir la imagen de la historia de la ciencia que presenta Kuhn sus críticos tuvieron que apelar a los conceptos kuhnianos. Pues no se encontraba disponible un marco conceptual tan rico como el propuesto por Kuhn para hablar de los aspectos diacrónicos de la ciencia.

En este trabajo discutiré la aplicación del concepto de inconmensurabilidad a una de las revoluciones científicas más radicales en lo que respecta a la forma en que cambió nuestra imagen del mundo: la darwiniana. Me interesa en particular discutir la presunta inconmensurabilidad existente entre la teoría de la selección natural de Darwin (en adelante TSND) y la teoría del diseño inteligente de la teología natural (en adelante DITN). ${ }^{2}$ Como es bien sabido el concepto de inconmensurabilidad incluye diferentes aspectos o fases. Sería posible e interesante discutir cada uno de estos aspectos al respecto de la revolución darwiniana, pero sólo me concentraré en la inconmensurabilidad empírica, es decir, discutiré la cuestión de si TSND y DITN pretenden o no dar cuenta del mismo tipo de fenómenos. De manera general, puede sostenerse, y suele sostenerse, que tanto TSND como DITN son teorías competidoras que intentan dar cuenta de la adaptación de los rasgos de los organismos vivos al ambiente. El tema del trabajo, entonces, consistirá en determinar si efectivamente el concepto de adaptación de Darwin es el mismo que el de los teólogos naturales. La respuesta no será por sí o por no. Hay sentidos en los que lo es, y sentidos en los que no, y la idea es desarrollar y discutir este punto.

Varias décadas han pasado desde los 60’ en donde esta discusión comenzó. El concepto de inconmensurabilidad (y otros conceptos kuhnianos) han sido retomados, elucidados y sofisticados en el marco del estructuralismo metateórico (Balzer et al. 1987). Asumiré tal marco a lo largo del trabajo.

En II presentaré la discusión existente acerca de si Darwin quiere dar cuenta de los mismos fenómenos de los cuáles los teólogos naturales querían dar cuenta (es decir, si

\footnotetext{
${ }^{1}$ Feyerabend (1962) también trata este problema apelando a la misma noción. Puede apelarse a Oberheim y Hoyningen-Huene (2013) para un tratamiento de las diferencias entre ambos autores y bibliografía al respecto.

${ }^{2}$ No estoy interesado en discutir en este artículo la naturaleza de las posiciones actuales que defienden el diseño inteligente como motor explicativo de la presencia de algunos o todos los rasgos de los organismos vivos, aunque la discusión precedente podría enriquecer la discusión al respecto de la naturaleza de tales posiciones.
} 
TSND y DITN tienen o no el mismo explanandum). En III introduciré el modo en que dentro del estructuralismo metateórico se piensa la inconmensurabilidad empírica. En IV presentaré la teoría subyacente a TSND con la que Darwin conceptualiza el explanandum de su teoría. En $\mathrm{V}$ presentaré la teoría subyacente con la que los teólogos naturales conceptualizan el explanandum de DITN. Con este fin esbozaré la ley fundamental de la teoría de la cual DITN sería una especialización y aclararé que la pregunta sólo tiene sentido al respecto de una parte de tal teoría. En VI compararé ambas teorías subyacentes y presentaré las modificaciones sustanciales que Darwin introdujo a la teoría subyacente a DITN desde la cual se conceptualizan las adaptaciones y, en consecuencia, al concepto de adaptación. Finalmente, en VII, discutiré si puede sostenerse que estas teorías subyacentes a las que tanto Darwin y como los teólogos naturales apelan, son o no la misma teoría. Esto me permitirá dar un veredicto al respecto de la cuestión de si TSND y DITN son o no empíricamente inconmensurables y me permitirá establecer con claridad en qué sentido Darwin revolucionó, además de la biología evolutiva, la biología funcional.

\section{La adaptación en la teología natural y en el darwinismo}

Darwin tenía en alta estima a la teología natural de Paley (Darwin 1958, 59), pero consideraba que los fenómenos que se podían explicar apelando a DITN eran ahora mejor explicados apelando a TSND (Darwin 1958, 87). Parece que bajo la perspectiva misma de Darwin éstas serían teorías incompatibles compitiendo por dar cuenta de un mismo tipo de fenómeno. Fenómeno al que tanto Darwin como Paley suelen referirse con el mismo término: 'adaptación'. ${ }^{3}$ De hecho cuando Darwin tiene que describir las adaptaciones en abstracto apela a las mismas características que Paley, y a las cuales se apela desde los orígenes mismo del argumento del diseño en los orígenes mismos de la filosofía: entidades complejas con partes coadaptadas que exhiben propósitos o fines (p.e. Hume [1779] 1980, 15; Kant [1781] 1998, A 626; Darwin 1859, 3, 60, 132; Paley 1809, 1-3). Por otra parte, forma parte de la historia oficial del darwinismo la idea de que Darwin logró dar una mejor explicación de aquello que era explicado por los teólogos naturales. En una primera mirada podría pensarse, en este sentido, que el concepto de adaptación, disponible con anterioridad a las teorías propuestas por Darwin, permitiría describir adecuadamente el ámbito de aplicación o el explanandum de TSND.

Ha sido señalado, sin embargo, que las adaptaciones darwinianas eran de otra naturaleza que las de la teología natural. Por ejemplo, Ospovat (1980) considera que para los teólogos naturales las adaptaciones, al estar diseñadas por un arquitecto perfecto, heredarían esta perfección de su creador. En cambio las adaptaciones darwinianas serían, dada la naturaleza mundana de la selección natural, imperfectas (o subóptimas). $\mathrm{Si}$ esto fuese cierto entonces no se podría sostener que TSND y DITN tienen el mismo explanandum.

\footnotetext{
${ }^{3}$ El concepto de adaptación es ambiguo, y es utilizado por Darwin ambiguamente. A veces se usa "adaptación" para hablar de lo que se quiere explicar con TSND y a veces se usa para hablar de rasgos que surgieron por selección natural (Ginnobili 2010a, 41). Aquí usaré la expresión en el primer sentido.
} 
Ospovat (1980) defiende dos tesis:

1. Aunque ya desde 1830 Darwin rechazaba las explicaciones teológicas sostuvo hasta 1854 que aquello que su teoría explicaba era la aparición y fijación de rasgos óptimos. En el Origen Darwin ya sostiene que la adaptación es imperfecta.

2. La creencia temprana en la perfección de las adaptaciones provenía de la influencia de los teólogos naturales.

Blanco (2008) discute la segunda de las tesis. Pues, cómo muestra convincentemente, los teólogos naturales de ningún modo consideraban que las adaptaciones de los organismos vivos fuesen perfectas. Los teólogos naturales del siglo XIX, en todo caso, hablaban de la perfección de la obra en general, pero esto no era incompatible con imperfecciones locales. Eran conscientes de éstas e intentaban dar respuestas desde sus sistemas teológicos. Es irrelevante en este caso que apelando a TSND se diera una mejor explicación de la imperfección de las adaptaciones, o bien en el sentido en el que la explicación de las retrogradaciones de los planetas de Copérnico es mejor que la de Ptolomeo (aunque el fenómeno es explicado por ambas teorías) o bien en el sentido en que Copérnico da una mejor explicación del hecho de que los planteas internos nunca se opongan al Sol (fenómeno no explicado, pero sí compatible con la teoría de Ptolomeo ${ }^{4}$ ). El punto en cuestión es que los fenómenos de los cuales se quería dar cuenta eran los mismos: adaptaciones con distintos grados de perfección (óptimas y subóptimas) (Blanco 2008, 20).

Para marcar el contrapunto con esta posición, apelaré a Caponi (2011). En el primer capítulo de su libro Caponi intenta derribar lo que caracteriza como "el mito del adaptacionismo predarwiniano”. Según Caponi, la forma en que Darwin concibió a la adaptación no se encontraba presente ni en teólogos naturales ni en ningún otro naturalista anterior.

En realidad, para que el interés central de los naturalistas se pudiese desplazar desde la correlación de forma y función en el interior del ser vivo, a la correlación entre los perfiles orgánicos y las exigencias del medio, y para que los antagonismos entre los seres vivos conquisten esa importancia que tienen en la historia natural darwiniana, era necesario que se operase una alteración radical en el modo de ver la naturaleza y en el modo de entender el lugar de los diferentes seres vivos dentro de ella. Esa alteración tiene que ver con la debacle de la idea clásica de economía natural. (Caponi 2011, 14-15)

Según esta idea de economía natural, la razón de un ser vivo y de sus rasgos reside en la manutención del orden natural general de la naturaleza, que en el caso de los teólogos naturales, dependía del plan de la creación. Darwin, además de revolucionar la biología evolutiva habría inaugurado una nueva forma de ver el mundo, distinta de la de sus antecesores, en la cual los organismos vivos hacen lo que hacen en búsqueda de su propio beneficio (entendiendo beneficio darwinianamente, por supuesto, en relación con mejoras en su éxito reproductivo diferencial). Este es el sentido de frases de

\footnotetext{
${ }^{4}$ Es decir, en la teoría de Ptolomeo que los planetas interiores no se opongan al Sol es una contingencia compatible con el sistema, mientras que en Copérnico es una consecuencia necesaria del sistema.
} 
Darwin que pueden parecer algo exageradas si no se tiene en mente el cambio gestáltico aludido:

Si se pudiese probar que una parte cualquiera del organismo de una especie ha sido formada para ventaja exclusiva de otra especie, esto destruiría mi teoría, pues esta parte no podría haber sido producida por selección natural. (Darwin 1859, 201)

Un ejemplo de esto lo constituiría la idea de que la belleza en los seres vivos ha sido creada para nuestro disfrute estético (Darwin 1872, 159-160).

El punto señalado por Caponi permite entender que estas dos citas tratan sobre el cambio operado en el modo de ver la naturaleza. En el marco de la teología natural era perfectamente aceptable y esperable que ciertos rasgos de los organismos sirvieran a otras especies (Paley 1809, 351) como también lo era la apelación a la belleza, con respecto a nuestros estándares o a estándares objetivos, para dar cuenta de ciertos rasgos de los organismos vivos (Paley 1809, 199-200).

Toda esta discusión arroja dudas al respecto del estatus de la inconmensurabilidad empírica entre las teorías que estamos tratando. Según Blanco nos encontraríamos frente a un caso de teorías empíricamente conmensurables, pues, ambas teorías quieren dar cuenta del mismo fenómeno: adaptaciones que no excluían la imperfección. Caponi, en cambio, señala que, perfectas o no, las adaptaciones de la teología natural serían de diferente tipo, puesto que incluirían adaptaciones antropocéntricas o al servicio de la economía natural, mientras que las adaptaciones darwinianas siempre tendrían que ver con metas del organismo que las porta. Según Caponi la revolución darwiniana incluiría un cambio en el modo en que el fenómeno del cual se quiere dar cuenta es conceptualizado, siendo TSND y DITN, en consecuencia, empíricamente inconmensurables. ¿Es posible tomar partido en esta polémica?

En la siguiente parte del trabajo presentaré de un modo más preciso en qué consiste la inconmensurabilidad empírica apelando a la presentación que de esta noción se hace desde el estructuralismo metateórico, para poder colaborar con la solución de esta disputa.

\section{La inconmensurabilidad empírica bajo el marco del estructuralismo metateórico}

Puede hablarse en dos sentidos de 'inconmensurabilidad empírica', y ambos sentidos pueden ser encontrados en la obra de Kuhn (Falguera 2012, 123-127). Por un lado, podemos referirnos a la inconmensurabilidad perceptual_de la que hablaba especialmente, aunque no exclusivamente, Hanson (1958, cap. 1). Científicos bajo diferentes paradigmas pueden organizar su percepción de diferentes modos, pudiendo ocurrir que aunque dirijan su mirada hacia lo mismo, tengan una percepción gestálticamente diferente. Por otro lado, la inconmensurabilidad empírica puede referirse a la inconmensurabilidad entre los lenguajes con los que se describen los fenómenos de los que se quiere dar cuenta desde las teorías en cuestión. El concepto de inconmensurabilidad empírica que nos interesa en este artículo es el segundo. El análisis que estamos intentando llevar adelante tiene que ver con los conceptos o el lenguaje a los que Darwin y los teólogos naturales apelan para hablar de los fenómenos que quieren explicar desde sus respectivas teorías. En adelante-y he venido hablando de este modo en todo el artículo-me referiré con 'inconmensurabilidad empírica' a este sentido en 
que dos teorías comparten o no el lenguaje con el que se categoriza su explanandum. Por supuesto, en este sentido la inconmensurabilidad se vuelve una cuestión de grado, puesto que es posible que las teorías difieran en algunos de sus conceptos (Díez 2012, 90-91). La inconmensurabilidad empírica, en el marco de la concepción clásica de teoría, en donde el explanandum de una teoría se describe con términos observacionales, se relaciona siempre con la carga teórica del lenguaje observacional. Pero, como veremos, desde el estructuralismo metateórico puede darse una versión más sofisticada de la inconmensurabilidad empírica, apelando a la distinción de $T$-teoricidad.

Los estructuralistas ofrecen una distinción entre los conceptos primitivos de una teoría sumamente fructífera y aclaratoria: la distinción entre conceptos $T$-teóricos y $T$ no teóricos. Esta distinción, a diferencia de la distinción clásica, no apela en absoluto a la observabilidad. Los conceptos $T$-teóricos de una teoría $T$ son aquellos cuya aplicación presupone en todos los casos a la teoría $T$, mientras que los conceptos $T$-no teóricos, pueden ser aplicados en prescindencia de $T$. La existencia de estos últimos permite que la contrastación de $T$ sea independiente, no de toda teoría-puesto que los conceptos $T$-no teóricos en $T$ pueden ser $T$-teóricos en otra/s teoría/s-pero sí de $T$. Los conceptos con los que se describe el fenómeno a explicar por una teoría son $T$-no teóricos, puesto que era posible aplicarlos antes de que $T$ existiera. Si $T$ explica el fenómeno apelando a otros conceptos, estos suelen estar propuestos por $T$ y su aplicación suele presuponer T. Esta distinción fue propuesta originalmente por Sneed (1971) y fue introducida en el marco del estructuralismo metateórico como la distinción informal de T-teoricidad (Balzer et al. 1987, 47-78).

Esta distinción, que influyó sobre la forma misma en que Kuhn consideraba la inconmensurabilidad (Lorenzano 2012, 302; Díez 2012, 67-72), permite caracterizar de un modo más preciso la noción de 'inconmensurabilidad empírica'. Dos teorías serían teóricamente inconmensurables si no comparten su vocabulario teórico (o parte de este). Dos teorías serían empíricamente inconmensurables, si apelan a diferentes conceptos para conceptualizar el fenómeno del cual quieren dar cuenta, es decir, cuando no comparten su lenguaje no teórico-o parte de éste (Lorenzano 2012, 308; Díez 2012, 71-72 y 92). ${ }^{5}$ En el modo estructuralista de hablar, dos teorías serían empíricamente conmensurables, en este sentido, si tienen los mismos modelos potenciales parciales-

${ }^{5}$ La idea de compartir lenguaje teórico y o no teórico esta presentada aquí de modo intuitivo. Tanto Lorenzano (2012), Díez (2012) como Falguera (2012) establecen distinciones más precisas y presentan discusiones más sofisticadas al respecto de la inconmensurabilidad. Aquí no presento más de lo que necesito para continuar y discutir el punto relevante al trabajo. Lorenzano no habla en el artículo citado de 'inconmensurabilidad empírica' sino de 'comparabilidad empírica'. Independientemente de las expresiones utilizadas la cuestión es la misma, tratar un sentido en el que puede discutirse si una teoría habla de lo mismo que otra o no. Finalmente, los autores citados, especialmente Díez y Falguera, señalan diferentes sentidos en los que podrían compararse teorías que no comparten su lenguaje no teórico aunque de todos modos existe un sentido en que 'hablan de lo mismo', como la mecánica newtoniana de partículas y la relativista. Tales consideraciones no vienen al caso en este trabajo. A su vez, el enfoque estructuralista de la inconmensurabilidad no considera la cuestión de la posibilidad de traducción (en la que Kuhn tenía un especial interés) sino meramente la cuestión—más manejablede compartir o no métodos de determinación de los conceptos (Díez 2012, 89-90). En este trabajo me restringiré a la noción presentada de inconmensurabilidad empírica y sólo sacaré conclusiones al respecto. 
recordando además que esta es una cuestión de grado, de acuerdo a los distintos componentes que pueden compartir.

Retomando, entonces, bajo este enfoque la base 'empírica' de una teoría se encuentra conceptualizada a partir de conceptos $T$-no teóricos. Una teoría da cuenta de los fenómenos de su base 'empírica' enriqueciendo la descripción previa no teórica con nuevos conceptos (usualmente T-teóricos). En este marco puede presentarse la cuestión de cuál es la base 'empírica' de una teoría $T$ dada, del siguiente modo: ¿a qué teorías previas y subyacentes apela $T$ para caracterizar los fenómenos que pretende explicar?6 $\mathrm{Y}$ para discutir si $T$ y $T$ son inconmensurables habría que responder a la pregunta: ¿apelan $T$ y $T$ a la misma teoría previa y subyacente para determinar de manera independiente los conceptos con los que se categorizan los fenómenos que quieren explicar? En el caso más sencillo de conmensurabilidad empírica, las teorías $T$ y $T$ ' apelarían a una y la misma teoría para conceptualizar sus respectivas bases 'empíricas'. Si este es el caso, por más que $T$ y $T$ ' sean teóricamente inconmensurables, su comparación sería menos problemática al compartir ambas la base de contrastación. Si bien es posible pensar, como decíamos, en diferentes grados de inconmensurabilidad a partir de los diferentes componentes compartidos, me restringiré en este trabajo a esta noción más restringida de inconmensurabilidad y sólo acerca de ella sacaré conclusiones.

Hemos presentado un marco para volver a la cuestión que nos compete más manejable. Veamos entonces, si TSND y DITN apelan o no a la misma teoría subyacente para determinar el concepto de adaptación, y, de no apelar a la misma teoría, qué relación podemos encontrar entre ambas teorías subyacentes, de modo de clarificar la cuestión de si TSND y DITN son empíricamente inconmensurables o no.

\section{La biología funcional darwiniana}

Cómo veíamos, lo que TSND pretende explicar son las adaptaciones de los organismos al ambiente. Además, vimos que la caracterización general y abstracta que Darwin hace de las adaptaciones no difiere de la caracterización que los defensores del argumento del diseño-y alguno de sus críticos-hacen de los objetos diseñados: complejos, con partes coadaptadas y con propósitos. Sin embargo, para entender con claridad el modo en que el concepto de adaptación es utilizado por Darwin, es necesario apelar a los casos particulares en los que lo aplica.

Si se revisan los diferentes fenómenos a los que Darwin quiere aplicar TSND puede notarse que apelan siempre a especificaciones de los mismos conceptos:

- ¿Cómo las jirafas adquirieron el cuello que les permite comer de las ramas altas de los árboles? (Darwin 1872, 177)

- ¿Cómo las perdices adquirieron el color que les permite mimetizarse con su ambiente? (Darwin 1859, 84-85)

\footnotetext{
${ }^{6}$ No quiero implicar aquí que todo concepto no teórico en una teoría se determina a partir de otras teorías. Esto no está claro. En el caso tratado, sin embargo, este es el caso, al menos, para los conceptos que nos incumben, como trataré de mostrar.
} 
- ¿Cómo las aves adquirieron el color del plumaje que les permite atraer a las hembras? (Darwin 1859, 88-89)

- ¿Cómo los gallos adquirieron espolones que les permiten luchar con otros gallos por las hembras? (Darwin 1859, 88)

- ¿Cómo las plantas adquirieron flores con la capacidad de segregar néctar y atraer a los insectos? (Darwin 1859, 92)

En todos estos casos, que sólo son algunos de los tratados por Darwin, lo que se pretende explicar es cómo un tipo de organismos ha adquirido un rasgo que cumple una función de manera altamente efectiva.

En otras oportunidades presenté reconstrucciones de TSND (Ginnobili 2009b; Ginnobili 2010a; Ginnobili 2010b; Ginnobili 2011a; Ginnobili 2012). Aquí baste decir que la forma en que Darwin explica el origen de la adaptación es apelando a iteraciones a lo largo de muchas generaciones de una teoría que tiene la siguiente ley fundamental (presentada informalmente):

Los organismos que portan rasgos que cumplen su función de manera más efectiva tienden a mejorar su aptitud, tendiendo a mejorar en consecuencia, de ser los rasgos en cuestión heredables, su éxito reproductivo diferencial.

No estoy aquí interesado en profundizar sobre este punto, puesto que este trabajo trata sobre la conceptualización del fenómeno del cual Darwin quiere dar cuenta. Baste decir que la explicación de Darwin del origen e incremento de la adaptación de los organismos al ambiente se explican apelando a esta ley fundamental (entre otras cosas), que generación tras generación incrementa el éxito reproductivo de los organismos cuyos rasgos cumplen comparativamente mejor su función. Como las variaciones suelen heredarse, generación tras generación, el grado de efectividad con la que se cumple tal función va incrementándose.

La cuestión relevante aquí es que la determinación de los fenómenos de los cuales Darwin quiere dar cuenta con TSND presupone tareas previas realizadas desde la biología funcional. La biología evolutiva darwiniana presupone su biología funcional. Afortunadamente para nuestras tareas reconstructivas, Darwin tiene una gran cantidad de textos cuya temática principal pertenece a la biología funcional.

Por supuesto, la discusión acerca de la explicación funcional y los conceptos funcionales es extensa y compleja. En otra oportunidad defendí cómo el estudio de la biología funcional darwiniana permite pensar nuevos modos de afrontar la polémica acerca de la explicación funcional (Ginnobili 2009a; Ginnobili 2011b). Aquí no tomaré en cuenta toda esta discusión. Simplemente presentaré la cuestión-siguiendo el trabajo realizado en los trabajos anteriormente mencionados_-del modo más natural para el que trabaja en el marco del estructuralismo metateórico: si un científico explica ciertos fenómenos apelando a ciertos conceptos, entonces, debe haber teorías-en el sentido del estructuralismo metateórico—en los que tales conceptos adquieran semántica empírica y que permitan reglamentar su aplicación. Olvidemos la biología evolutiva, por un momento, y veamos entonces, aunque sea informalmente, la estructura de la teoría subyacente a TSND con la que se categoriza el fenómeno de la adaptación. Me referiré a tal teoría como 'teoría funcional biología darwiniana' (en adelante TFBD). 
No hay textos de Darwin más claros al respecto de cómo funciona su biología funcional que aquellos que versan sobre la fecundación cruzada en el reino vegetal (Darwin 1861; Darwin 1876; Darwin 1877b; Darwin 1877a). Pues es en estos textos donde Darwin intenta señalar que el evitar la fecundación cruzada es la meta—por supuesto no consciente-que muchos de los rasgos de las flores persiguen. Sin tener en cuenta esta función, las estructuras florales serían completamente incomprendidas.

Tómese en consideración el siguiente párrafo:

Aquí entra en juego de nuevo otra bonita adaptación [...] [de la Orchis pyramidalis]. El estigma es muy viscoso; no tan viscoso como para que al ser tocado por un polinio pueda arrancar la cabeza completa de un insecto o un lápiz, pero sí lo suficientemente viscoso para romper las hebras elásticas que unen los paquetes de granos de polen y dejar algunos sobre el estigma. Por consiguiente, un polinio adherido a un insecto o a un lápiz puede aplicarse a muchos estigmas y los fecundará a todos. (Darwin 1877b, 13-14)

En esta pequeña explicación de la estructura floral de esta especie de orquídeas se apelan a varios conceptos:

- Organismos de cierta tipo: Orchis pyramidalis

- Rasgos con ciertas características: estigma muy viscoso

- Efectos que estos rasgos tienen: si uno toca el estigma con un polinio adherido a un lápiz o si un insecto lo toca con un políneo adherido a su probóscide, no se pega a este, pero rompe las hebras elásticas que unen los paquetes de grano de polen, y algo de este cae sobre su estigma.

- Objetivos: facilitar la fecundación por otras orquídeas de la misma especie.

Algunos de estos conceptos pertenecen a un nivel de descripción estructural o comportamental-organismos de cierto tipo, rasgos y efectos. La forma en la que la biología funcional darwiniana da cuenta de tales estructuras y comportamientos es apelando al concepto de meta u objetivo, en este caso en particular, al concepto de facilitar la fecundación cruzada, o evitar la autofecundación.

La ley fundamental de $\mathrm{TFB}^{\mathrm{D}}$, en la que deberíamos encontrar relacionados todos estos conceptos, serviría de guía a los biólogos para encontrar los objetivos de los organismos satisfechos por ciertos efectos privilegiados de ciertos rasgos en ciertas condiciones ambientales. Por supuesto, si bien se puede afirmar que todo rasgo tiene efectos bajo ciertas condiciones, no se puede afirmar que todo rasgo cumpla con algún objetivo, es decir, que todo rasgo tenga una función. Lo que sí podemos saber es que si un organismo tiene cierto objetivo-reproducirse, sobrevivir, alimentarse-, entonces tiene que tener rasgos, al menos uno, cuyos efectos satisfagan ese objetivo en ciertas condiciones ambientales. Si un organismo no tuviera ningún rasgo que satisficiera un objetivo, al menos en algún ambiente, entonces no tendría sentido sostener que tal organismo tiene ese objetivo. La ley fundamental podría afirmar, en consecuencia, algo como lo siguiente:

Para todo organismo, si el organismo tiene cierto objetivo, ciertos efectos de alguno de sus rasgos en ciertas condiciones ambientales satisfacen tal objetivo.

No creo que sea posible afirmar nada más fuerte que esto o dar más información que la dada. No se puede afirmar que la posesión del rasgo sea condición ni necesaria 
ni suficiente para cumplir el objetivo como ha sido señalado en reiteradas ocasiones en las discusiones acerca de las funciones. ${ }^{7}$ La dirección del condicional en la ley fundamental permite captar este salto abductivo inherente a la postulación de un nuevo objetivo para un organismo. El único modo viable de sostener que cierto organismo persigue la meta de evitar autofecundarse, por ejemplo, es encontrando una serie de rasgos cuyos efectos satisfagan esa meta. La forma en que Darwin muestra que las plantas tienen la meta de evitar autofecundarse, es mostrando la serie de rasgos cuyos efectos persiguen tal meta a lo largo de todo el reino vegetal.

Habitualmente cuando en la práctica científica se habla de teorías, se refiere a lo que dentro del estructuralismo se nombra como "redes teóricas" (Balzer et al. 1987, 167-204). Una red teórica es un conjunto de elementos teóricos que surgen por especialización del elemento teórico básico. Las especializaciones del elemento teórico básico son el equivalente modelo-teórico a las leyes especiales. Estas surgen, no por deducción, sino por especificación de conceptos que en el elemento teórico básico se encuentran indeterminados.

Considero que las leyes especiales de TFBD surgen principalmente de la especificación del concepto de objetivo, es decir, las diferentes leyes especiales surgen de los diferentes tipos de objetivos que pueden satisfacer los rasgos de los organismos. Algunas de las especificaciones diferentes, o especializaciones de esta ley fundamental, serían las siguientes - los ejemplos no son elegidos arbitrariamente, sino que son algunos de los muchos que trata el mismo Darwin:

- El largo del cuello de la jirafa tiene la función de alcanzar las ramas altas de los árboles para alimentarse en los períodos de escasez (Darwin 1872, 177).

- La forma y el color de la cola del pavo real tiene la función de atraer a las hembras para reproducirse (Darwin 1859, 88-89).

- Los espolones de los gallos tienen la función de luchar con otros gallos por las hembras para reproducirse (Darwin 1859, 88).

- El color de ciertos insectos tiene la función de mimetizarse y defenderse de los predadores (Darwin 1872, 182).

- El aguijón de las abejas tiene la función de defender el panal en el que viven (Darwin 1859, 202).

Las diferentes especializaciones de la ley fundamental pueden ser agrupadas en diferentes clases de acuerdo al tipo de función de que se trate. Así, muchas funciones tienen que ver con la reproducción, otras con la defensa de predadores, otras con la alimentación, etc. Podríamos pensar que estos diferentes tipos de explicaciones representan justamente, aplicaciones de diferentes leyes especiales que sirven de guía a Darwin a la hora de aplicar TFBD. Los siguientes podrían ser ejemplos de diferentes leyes especiales a partir de los casos citados:

\footnotetext{
${ }^{7}$ Sirva de ejemplo la famosa discusión acerca de los equivalentes funcionales.
} 
- Para todo organismo, si el organismo tiene el objetivo de alimentarse, ciertos efectos de alguno de sus rasgos en ciertas condiciones ambientales satisfacen tal objetivo.

- Para todo organismo, si el organismo tiene el objetivo de defenderse de los predadores, ciertos efectos de alguno de sus rasgos en ciertas condiciones ambientales satisfacen tal objetivo.

- Para todo organismo, si el organismo tiene el objetivo de reproducirse, ciertos efectos de alguno de sus rasgos en ciertas condiciones ambientales satisfacen tal objetivo.

La lista no es fija. Como suele ocurrir en la historia de las teorías científicas, durante el desarrollo de la teoría se descubren nuevas leyes especiales. En el marco de la defensa de una nueva ley especial de $\mathrm{TFB}^{\mathrm{D}}$ creo que es en donde hay que colocar los escritos de Darwin dedicados a la importancia y extensión de la fecundación cruzada. En la Figura 1 presento una propuesta de reconstrucción de la red teórica de TFBD considerando diferentes metas a las que Darwin apela en su rol de biólogo funcional con la rama de la fecundación cruzada completa, que es la que mejor se desarrolla en sus textos sobre la fecundación cruzada (Ginnobili 2011b).

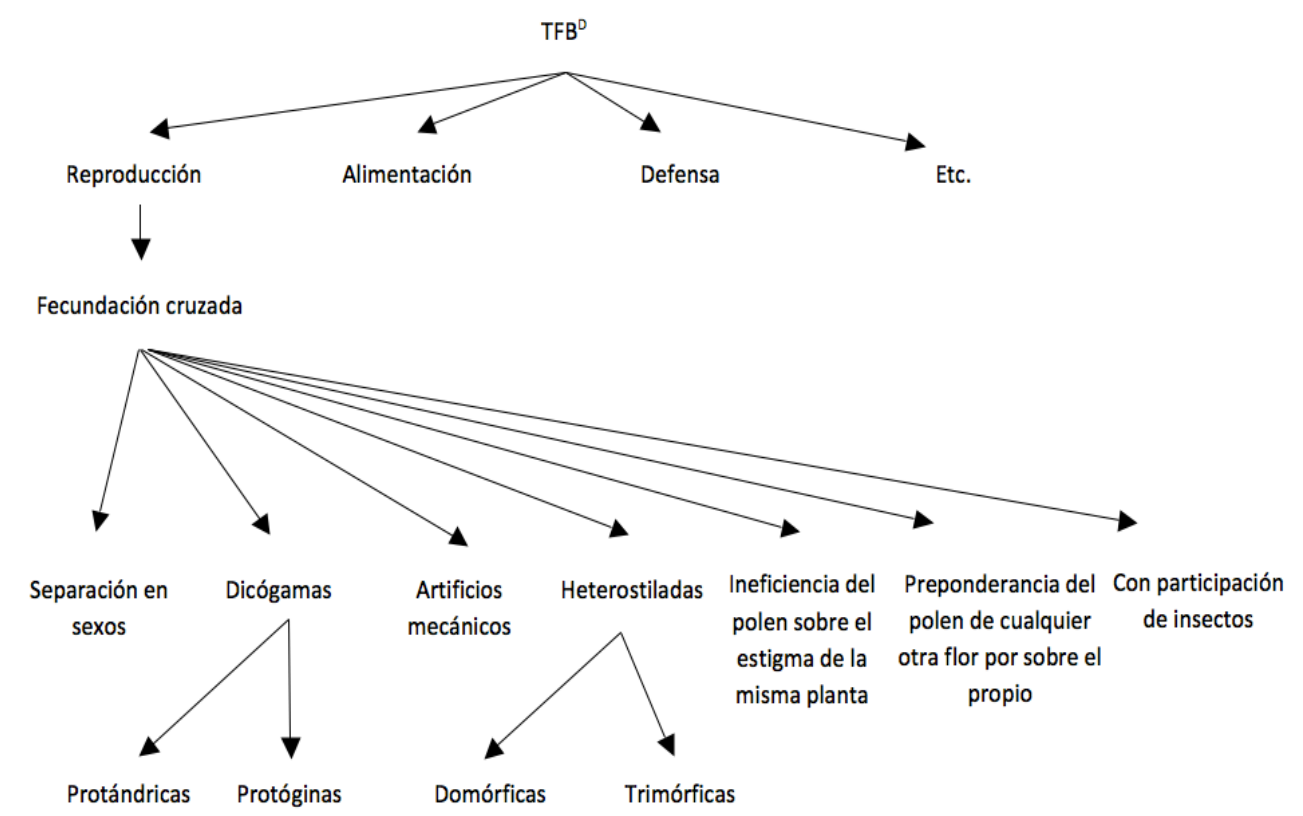

Figura 1: Red teórica de TFBD con la rama del favorecimiento de la fecundación cruzada desplegada de la red teórica de TFB. 


\section{La biologia funcional de la teología natural}

Para discutir con sentido si DITN acude o no a la misma teoría subyacente que TSND hay que tener en claro que la teoría del diseño inteligente en general (DI en adelante) tiene un alcance más amplio que TSND. DI es una teoría que se aplica de un modo general a artefactos explicando los rasgos funcionales de una entidad en base a los objetivos conscientes del sujeto particular inteligente que los creo. Así, los objetivos perseguidos por diversos rasgos de ciertos objetos-entre los cuales para los teólogos naturales se encontrarían los rasgos funcionales de los organismos-son subsumidos o reinterpretados como objetivos conscientes del diseñador. Este es el modo en que lo plantea el mismo Paley en su presentación del argumento del diseño (Paley 1809, 1-3).

La fuerza del argumento consiste en señalar que si uno acepta la explicación de los rasgos de un reloj a través de la postulación de un diseñador inteligente, lo mismo debería hacer con los rasgos de los organismos vivos.

Podemos pensar que la ley fundamental de la teoría del diseño generalizada afirma algo como lo que sigue:

Si un sujeto con ciertos objetivos conscientes diseña un artefacto para satisfacer dichos objetivos, entonces, ese artefacto tendrá rasgos que cumplen con esos objetivos.

La dirección del condicional refleja también en este caso el salto abductivo necesario para postular diseñadores y postular los objetivos que persiguen. El argumento del diseño, justamente, sigue esa dirección. Esta teoría es utilizada todo el tiempo, por ejemplo, al inferir que las melladuras de ciertas piedras no son aleatorias, sino que fueron realizadas, por ejemplo, por homo erectus con el objetivo de producir una herramienta para cortar. También permite explicar, por ejemplo, la posesión de ciertas características de la soja que hoy se comercializa, obtenidas por el diseño genético (no por selección artificial). La fuerza del argumento del diseño radica en que se sustenta sobre la aplicación de una teoría que todos aceptamos, como bien señala Paley. Por ese motivo desde el comienzo de este trabajo tomé como objeto de la discusión la teoría del diseño de la teología natural, que podría considerarse una especialización (que hoy se ha abandonado por buenas razones) de la teoría DI. ${ }^{8}$ Así, estamos comparando una teoría (TSND) con una parte de una teoría (DITN).

Pero, además, debemos hacer un recorte más para que la cuestión tenga sentido. Puesto que los teólogos naturales aplicaban DI ${ }^{\mathrm{TN}}$ a objetos a los que Darwin no pretendía de ningún modo aplicar TSND. Por ejemplo, Paley no duda en sostener que la noche es una adaptación al sueño de los animales (Paley 1809, 295). Esto es perfectamente aceptable en el marco de la teología natural. Así como el fruto es un artefacto diseñado con la función de alimentar a los animales, la noche tendría la función de favorecer un sueño tranquilo. La noche y otros objetos inanimados pueden caer bajo la aplicación tanto de DITN como de su teoría funcional subyacente.

\footnotetext{
${ }^{8}$ Aunque habría que discutir más pormenorizadamente si DITN es una especialización de DI o si entre estas teorías hay algún otro tipo de relación. Definir esta cuestión si bien es interesante para una reconstrucción de DI y de DITN, no es necesario para los fines de este trabajo.
} 
Para que la discusión tenga sentido, entonces, nos restringiremos a DITN cuando se pretende aplicar a rasgos de los organismos vivos, pues sólo de esa parte tiene sentido preguntarse si es empíricamente inconmensurable con TSND. Aclarando sin embargo que DITN tiene un campo de aplicación más amplio, al pretender explicar rasgos de entidades o procesos no vivos. Seguiré hablando de DITN sin cualificaciones para no traer más confusión, para referirme a esta porción relevante de la teoría. Esto implica también un recorte en la teoría subyacente presupuesta, puesto que la noche sería una adaptación al sueño, los rasgos de la noche también podrían ser considerados funcionales en el mismo sentido de los rasgos de los organismos. En este caso también, sólo consideraremos la biología funcional de la teología natural (en adelante TFB $^{\mathrm{TN}}$ ) aplicada a organismos vivos para compararla con TFBD.

Tal como afirma Caponi $(2011,12)$, en los teólogos naturales existe una tendencia a focalizarse en las adaptaciones fisiológicas. Aquellas que tienen que ver con la economía animal o vegetal propia. Por ejemplo, podemos tomar el tratado quinto de la serie de libros que componen The Bridgewater treatises escrito por Roget (1834). En el segundo capítulo del primer volumen se cita de manera explícita el tipo de objetivos que pueden perseguir las diferentes partes de los organismos vivos, y que según él, por supuesto, permiten inferir la mano del diseñador. Así, las diferentes funciones que pueden atribuirse a los organismos vivos pueden dividirse en aquellas que tienen que ver con la percepción, aquellas que tienen que ver con el movimiento, las funciones mecánicas_que tienen que ver con el funcionamiento de la economía animal_, las funciones nutritivas-aquellas que tienen que ver con la alimentación-, y las funciones reproductivas (Roget 1834, 34-58). En mi interpretación de la biología funcional como la aplicación de una teoría, TFBTN, éstas bien podrían ser consideradas especializaciones del núcleo central presentado en el apartado anterior. Por supuesto, el libro de Roget trata justamente del apoyo de la fisiología a la teología natural, por lo que es esperable que sólo se consideren este tipo de funciones. Considerando sólo estas adaptaciones fisiológicas, la teoría con la que los teólogos naturales detectan funciones no parece diferir en lo sustancial de aquella utilizada por Darwin. La teoría aplicada por los teólogos naturales-en tanto biólogos funcionales-en lo que respecta a funciones fisiológicas no difiere de la aplicada por Darwin-en tanto biólogo funcional.

También coinciden en lo que respecta a ciertas aplicaciones que tienen que ver con relaciones con otros organismos que podríamos llamar 'ecológicas'. Cómo por ejemplo, Paley considera que ciertas partes de semillas que les permiten planear en el aire se relacionan con el objetivo que tienen las plantas de esparcir sus semillas (Paley 1809, 355), la luz de la luciérnaga tiene la función de atraer a la pareja reproductiva (Paley 1809, 336) e incluso también la cantidad de hijos es vista como una adaptación (Paley 1809, 481). Tres fenómenos que Darwin explícitamente discute como adaptaciones (Darwin 1844, 92; Darwin 1871, 345; Darwin 1859, 66).

Las diferencias importantes aparecen en otras ramas de la teoría. Aquellas que tienen que ver con el mantenimiento de la economía natural-tal como vimos que señala Caponi-, aquellas que involucran el antropocentrismo de la creación y aquellas que involucran objetivos estéticos como un fin en sí mismo. 
Por ejemplo, con respecto a funciones cuyo objetivo es el mantenimiento de la economía natural, puede mostrarse la opinión de Paley respecto de los frutos:

De la sola conformación de la fruta, uno puede ser conducido, incluso sin experiencia, a suponer que parte de su provisión fue destinada para la utilidad de los animales. Limitada a la planta, la provisión parece ir más allá de su objeto. [...] Los eventos muestran que esta redundancia, si la hubiera, atiende al soporte y gratificación de las naturalezas animales. (Paley 1809, 351)

La utilidad de los frutos tiene que ver justamente con la alimentación de los animales-y no sólo de la semilla_, es decir, se comprende un fruto a partir del rol que este cumple en la economía natural. Otro ejemplo podría constituirlo el rol de las plantas como purificadoras del ambiente (Paley 1809, 372).

Con respecto a la belleza como un fin en sí mismo, Paley sostiene:

¿Por qué, por tomar un ejemplo de muchos, la corola del tulipán, cuando avanza en su tamaño y madurez, cambia su color? El propósito, hasta donde podemos ver, de la nutrición vegetal, podría haber sido mantenido también si continuara siendo verde. [...] ¿No es más probable que esta propiedad, que es independiente, como parece ser, de las necesidades y utilidades de la planta, haya sido calculada para la belleza, procurada para la ostentación? (Paley 1809, 199-200)

La belleza a veces es planteada como un fin de la creación en sí mismo y otras parece funcionar al servicio de lo humanos (Paley 1809, 202) o de otros congéneres (Paley 1809, 197). Ejemplos, entre otros, de la función estética lo constituyen el plumaje adornado de las aves o el color del iris (Paley 1809, 198-199).

Con respecto a la visión antropocéntrica de las funciones podemos citar el siguiente ejemplo:

El omnisciente gobernador del universo, cuando le dio a la oveja su cubierta, parece que hubiera tenido en mente no sólo la protección del animal de los efectos del frío, sino más particularmente el beneficio de aquel que entronó en el centro de su creación, al poner a su disposición un material inestimable, por su utilidad y confort como lana. (Kirby 1836, 34-35)

La estructura de la red teórica que conforma TFBTN_ no completa, sino tomando sólo los casos mencionados-, en base a los diferentes tipos de objetivos perseguidos por los diferentes rasgos, sería tal como se muestra en la Figura 2. Las líneas punteadas llevan a casos en los que la ley especial se aplica-señalo sólo algunos porque me interesan especialmente.

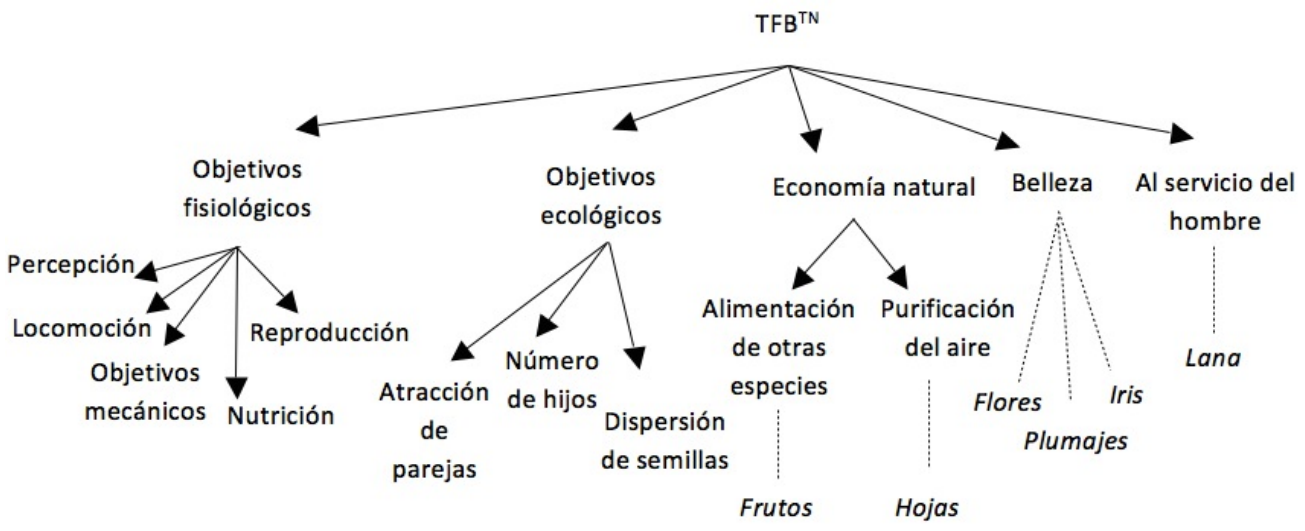

Figura 2: Red teórica de TFB ${ }^{T N}$. 


\section{Diferencias entre la biología funcional darwiniana y de la teología natural}

El análisis de $\mathrm{TFB}^{\mathrm{D}}$ presentado en la Figura 1 no es muy completo, porque se basa más que nada en la parte de la teoría que trata con la fecundación cruzada. Sin embargo, creo que es claro en qué sentido un mundo moldeado por la selección natural no sería compatible con la biología funcional de la teología natural. TSND es utilizada por Darwin para explicar el origen de las adaptaciones. Las adaptaciones son rasgos que cumplen una función de manera efectiva. Pero TSND sólo puede explicar el origen de funciones relacionándolas con beneficios reproductivos del propio organismo-o, eventualmente, con beneficios reproductivos del grupo al que el organismo pertenece, puesto que Darwin acepta la selección de grupo. Nunca podría explicar el origen de rasgos altruistas en beneficio de otra especie ni en beneficio de un sistema general de economía natural. Por este motivo, Darwin debe cambiar drásticamente la biología funcional. En particular, debe eliminar las especializaciones que apelan a roles en la economía natural, las que apelan al antropocentrismo de la creación y las que apelan a valores como la belleza o bondad de la creación. Como veíamos, las afirmaciones de Darwin acerca de cómo la constatación de que un rasgo sirva a otra especie sin beneficio a cambio refutaría su teoría, van justamente en ese sentido (Darwin 1859, 201). Darwin veía con claridad que era necesario cambiar la biología funcional presupuesta por la teología natural para que TSND cumpliera el rol requerido. A esta tarea es a la que avocó una enorme cantidad de tiempo y energías.

La eliminación de estas ramas de la red teórica de TFB ${ }^{\mathrm{TN}}$ no podía llevarse adelante sin más. En algunos casos podría eliminarse el fenómeno en cuestión del campo de aplicaciones pretendidas de la teoría, sosteniendo que en realidad el rasgo en cuestión no es funcional. El comportamiento o el rasgo citado sería un mero efecto de otra actividad funcional o no funcional. Por ejemplo, la purificación del aire provocada por las hojas de las plantas podría ser un efecto colateral de una actividad relacionada en realidad con una actividad fisiológica como la respiración de la propia planta. Pero tomar esta decisión con todos los fenómenos que bajo TFB ${ }^{\mathrm{TN}}$ eran explicados exitosamente, habría implicado una perdida costosa para la capacidad explicativa de la teoría. Los fenómenos que bajo TFB ${ }^{T N}$ eran explicados a través de las leyes que debían rechazarse, debían ser acomodados, entonces, bajo otras leyes especiales. Muchas de las discusiones de Darwin en biología funcional deben entenderse en este sentido.

Una estrategia de Darwin consiste en subsumir los fenómenos que ahora quedan sin explicar bajo ramas ya existentes en TFBTN. Por ejemplo, algunos de los rasgos explicados bajo TFB ${ }^{\mathrm{TN}}$ apelando al embellecimiento del mundo, serán subsumidos ahora bajo la rama ya existente que toma en cuenta el objetivo de atraer parejas reproductivas. Así, el plumaje de los machos de los pájaros (Darwin 1859, 88), o los colores del iris (Darwin 1871, v. II, 72), se subsumirán bajo el objetivo ecológico ya existente en TFBTN de atracción de parejas reproductivas. Tales objetivos serán subsumidos subsiguientemente en el enfoque evolutivo darwiniano apelando a la selección sexual, que puede pensarse como un caso de TSND (Ginnobili 2011c).

Lo mismo ocurre con casos que caen bajo la rama de especializaciones que toma en cuenta el rol de los organismos en la economía natural. Por ejemplo, el caso citado de los frutos, que Paley explica a partir del rol en el sistema de la economía natural de 
alimentar a los animales, será subsumido por Darwin bajo la rama preexistente que tiene como objetivo el esparcimiento de las simientes (Darwin 1844, 92). Algo semejante podrá hacerse con el ejemplo citado de la lana de la oveja, puesto que la función fisiológica de la lana no es problemática en absoluto.

Un caso más interesante lo constituye el de las flores, que también era explicado apelando a la belleza, para el cual Darwin debió crear una nueva ley especial en TFBD a partir del nuevo objetivo que tiene que ver con los beneficios de la fecundación cruzada. Mucho ha sido escrito sobre el comentario que Darwin hace a Asa Gray en una carta:

Nadie más se ha dado cuenta de que el objetivo principal de mi libro sobre las orquídeas ha sido un 'movimiento de flanco' sobre el enemigo. (Darwin 1862)

Este libro parece, bajo este punto de vista, supeditado al objetivo general de convencer a la comunidad científica de las tesis sostenidas en el Origen. Son muchos autores los que tratan distintos aspectos de esta cuestión (p.e. Ghiselin 1969, cap. VI; Beatty 2006; Lennox 1993; Gould 1980, cap. 1). En el marco presentado, el movimiento de flanco puede interpretarse como el intento de Darwin de defender sus puntos de vista evolutivos atacando no desde el frente (cosa que había hecho en el Origen a partir de evidencias convergentes) sino a través de un movimiento de flanco, cambiando la biología funcional subyacente aceptada hasta entonces.

\section{VII. ¿Son la teoría funcional de Darwin y de la teología natural etapas de una y la misma teoría?}

El trabajo realizado hasta el momento permite precisar y elucidar el sentido, señalado por Caponi, en que las adaptaciones tal como eran concebidas por los teólogos naturales no son las mismas que las adaptaciones tal como eran concebidas por Darwin. TFB $^{\mathrm{D}}$ es diferente en muchos aspectos de TFB ${ }^{\mathrm{TN}}$. Pero la pregunta acerca de la inconmensurabilidad empírica—en el sentido presentado anteriormente—entre TSND y DITN no queda respondida con sólo ese señalamiento. La cuestión es si hay un sentido interesante en el que el fenómeno de la adaptación sea el mismo, aunque haya sufrido los cambios que sufrió. En el marco planteado, la cuestión podría plantearse del siguiente modo. ¿Puede considerarse que $\mathrm{TFB}^{\mathrm{D}}$ y $\mathrm{TFB}^{\mathrm{TN}}$, aunque no sean idénticas, son diferentes momentos de la misma teoría - en el sentido diacrónico de teoría? Si sí, entonces, hay un sentido relevante en que TSND y DITN dan cuenta del mismo fenómeno o del mismo tipo de fenómenos.

El estructuralismo metateórico heredó de Kuhn el interés por el cambio teórico. No sólo se ofrece un marco conceptual para pensar teorías en un momento dado, sino que se pretende hablar también de la persistencia de teorías a lo largo del tiempo y los tipos de cambio que estas pueden sufrir. Una 'red teórica' sería entonces una teoría en un momento dado. Una 'evolución teórica' sería la teoría que sufre cambios a lo largo del tiempo-análogo al concepto de paradigma de Kuhn o de programa de investigación científica de Lakatos. Una evolución teórica sería, justamente, una serie de redes teóricas. Tal como señala Kuhn, existen dos tipos de cambios: cambios normales, en donde lo sustancial no se cambia, y cambios revolucionarios, en donde se abandona el paradigma. En el marco estructuralista, existen cambios que nos mantienen dentro de una misma evolución teórica, y existen cambios que implican que se inaugura una 
nueva evolución teórica. La pregunta que debemos responder sería entonces ¿es el cambio de Darwin en la biología funcional revolucionario o se trata de un cambio normal?

En el estructuralismo metateórico se citan los siguientes criterios para distinguir el cambio revolucionario del normal (Lorenzano 2012). Dos teorías serían casos de una misma teoría si:

1. Tienen el mismo marco conceptual. Utilizan los conceptos presentes en la ley fundamental sin agregar ningún concepto nuevo.

2. Tienen las mismas leyes fundamentales—aunque las leyes especiales pueden no ser las mismas.

3. Conceptualizan los fenómenos mediante los mismos conceptos.

4. Mantienen las mismas relaciones esenciales con otras teorías.

5. Tienen la intención de dar cuenta de al menos alguno de los mismos fenómenos.

Según lo que hemos venido viendo, y considerando los elementos que hemos señalado de ambas teorías-reconstrucciones más completas podrían llevarnos tal vez a considerar cuestiones que no hemos considerado, como relaciones esenciales con otras teorías-parece que las cinco condiciones se cumplen. Considerando estos criterios, podríamos considerar que $\mathrm{TFB}^{\mathrm{TN}}$ y $\mathrm{TFB}^{\mathrm{D}}$ son distintos momentos de la misma teoría.

La clave de la cuestión se encuentra, sin embargo, en la afirmación que aparece en el requisito 2 de que las leyes especiales pueden no ser las mismas. Puesto que un caso exagerado en que en momentos subsiguientes una teoría cambia todas sus leyes especiales, aunque inverosímil, difícilmente pueda considerarse como un cambio normal. Sería extraño considerar que esta nueva teoría sigue siendo la misma teoría, y que los conceptos propios y propuestos por la teoría siguen siendo los mismos. Por otra parte, el abandono de alguna ley especial de bajo nivel o el descubrimiento de una nueva ley especial de bajo nivel, no pueden ser considerados como cambios revolucionarios y no parecen afectar el significado de los conceptos fundamentales de la teoría. De hecho, una de las formas en que se aplica una ley fundamental a nuevos casos es justamente ésta, proponiendo nuevas leyes especiales. El caso de Darwin no se encuentra en ninguno de estos extremos. El cambio que realiza en la biología funcional parece más drástico que lo que habitualmente es considerado ciencia normal, pero mantiene ramas enteras de especializaciones.

Tal vez pueda ser útil apelar a lo afirmado por Díez (2002), quien ha señalado la necesidad de considerar ciertas leyes especiales de las ramas principales como constitutivas de la teoría. El ejemplo que trata es el de la mecánica clásica. Su punto sería que la fuerza de gravedad decrezca según el cuadrado de la distancia, también sería constitutivo del significado del concepto de fuerza (Díez 2002, 25-29). Si así fuera, la especialización principal en la que se especifica el concepto de fuerza como fuerza de gravedad, parecería esencial a la mecánica clásica. Si esta ley especial cambiara, podría considerarse que la mecánica clásica ha cambiado. Sin embargo Díez no brinda herramientas para establecer cuáles son las especializaciones constitutivas y cuáles no, sino 
que señala que el concepto de identidad conceptual es vago-aclarando que eso no implica que no pueda tratarse desde alguna herramienta metateórica. Si Díez tiene razón, la noción de cambio revolucionario también sería vaga. Esto implicaría que no pueda haber una respuesta univoca a la cuestión de si TFBD y TFB ${ }^{\mathrm{TN}}$ son o no la misma teoría, y por lo tanto, que no puede haber una respuesta unívoca al respecto de la inconmensurabilidad empírica entre TSND y DITN.

Si focalizamos en la continuidad, como lo hace Blanco en el trabajo antes mencionado, existen un sentido relevante en el que TSND y DITN son empíricamente conmensurables, y hablan de, en un sentido relevante e importante, los mismos fenómenos. Pues ambas teorías apelan a teorías subyacentes que comparten, en un sentido relevante, un mismo marco conceptual, una misma ley fundamental, un conjunto importante de leyes especiales y un conjunto importante de aplicaciones pretendidas y exitosas. Esto podría haber servido de base a la comparación que los contemporáneos a Darwin realizaron, y al triunfo del darwinismo sobre la teología natural. Si nos focalizamos en la ruptura, como lo hace Caponi, los cambios establecidos por Darwin en la biología funcional son más fundamentales que los cambios que habitualmente los científicos hacen en sus teorías en períodos de ciencia normal. Darwin elimina ramas principales completas de $\mathrm{TFB}^{\mathrm{TN}}$, abandona algunas de sus aplicaciones pretendidas, da cuenta de algunas otras a través de especializaciones disponibles, y propone nuevas especializaciones para dar cuenta de otras aplicaciones pretendidas. La tarea de Darwin en este sentido resulta más colosal y heroica, y la revolución darwiniana resulta más radical. Pues Darwin no sólo revolucionó la biología filogenética, sino que, además-y esto habitualmente no es reconocido—revolucionó la biología funcional.

\section{Conclusiones}

En este trabajo he intentado discutir la inconmensurabilidad empírica entre el enfoque darwiniano y el de la teología natural. En la discusión me he restringido únicamente, a una noción bastante fuerte de inconmensurabilidad empírica en donde dos teorías son empíricamente conmensurables sólo si dan cuenta del mismo tipo de fenómenos. $\mathrm{He}$ planteado la discusión en base al modo de tratar la cuestión de los estructuralistas metateóricos, en el que dos teorías hablan de los mismos fenómenos si comparten sus conceptos T-no teóricos. Si bien en este sentido puede haber diferentes grados de inconmensurabilidad, de acuerdo a los distintos componentes compartidos, para el caso en cuestión no ha sido necesario considerar estos matices. El punto en discusión es si Darwin y los teólogos naturales apelan o no a la misma teoría para categorizar el fenómeno del cual pretenden dar cuenta desde TSND y DITN respectivamente. Incluso considerando todas estas restricciones, la respuesta a la que hemos arribado es compleja, y tal vez, esta respuesta sea la única posible. Asumir una distinción más fuerte y menos vaga al respecto de la identidad de las teorías nos brindaría la ilusión de una solución a la cuestión de la inconmensurabilidad empírica-en el sentido señalado-entre los enfoques de Darwin y de los teólogos naturales, menos rica que la respuesta a la que hemos arribado, pues supondría que la historia de la ciencia es un fenómeno más simple de lo que realmente es. Independientemente de la cuestión terminológica al respecto de si podemos o no llamar 'revolucionario' al cambio producido 
por Darwin en la biología funcional, y en consecuencia, si podemos o no considerar éste un caso de inconmensurabilidad empírica—cuestión que en este trabajo ha quedado indeterminada—las herramientas del estructuralismo metateórico nos han permitido elucidar las intuiciones de los autores que se han acercado a la cuestión historiográficamente, y dar una descripción más pormenorizada de las relaciones entre las teorías biológicas funcionales subyacentes a las teorías filogenéticas en competencia. Esto permitirá abordar de manera menos ingenua la cuestión subsiguiente que existe al respecto de la comparabilidad entre ambos enfoques. ${ }^{9}$

Las aireadas disputas epistemológicas que surgieron alrededor del estudio de las revoluciones científicas no pueden resolverse a priori. Debemos dedicarnos a examinar estos episodios históricos, elaborar marcos conceptuales que nos permitan pensarlos, poniendo en cuestionamiento nuestras ideas epistemológicas previas acerca de la ciencia. Tal es la enseñanza más importante que uno puede extraer de la lectura de Kuhn. El esfuerzo no puede ser menos que gratificante, por los resultados a obtener, y además, porque no hay tarea más enriquecedora que la lectura de los escritos en los que se plasman los esfuerzos conceptuales de los que pensaron osadamente.

\section{REFERENCIAS}

Balzer, Wolfgang, Carlos U. Moulines y Joseph D. Sneed. 1987. An architectonic for science: the structuralist program. Dordrecht, Lancaster: Reidel.

Beatty, John. 2006. Chance Variation: Darwin on Orchids. Philosophy of Science 73 (5): 629-641.

Blanco, Daniel. 2008. La naturaleza de las adaptaciones en la teología natural británica: análisis historiográfico y consecuencias metateóricas. Ludus Vitalis XVI (30): 3-26.

Caponi, Gustavo. 2011. La segunda agenda darwiniana. Contribución preliminar a una bistoria del programa adaptacionista. México: Centro de estudios filosóficos, políticos y sociales Vicente Lombardo Toledano.

Darwin, Charles. 1844. Essay of 1844. En The foundations of The origin of species - Two essays written in 1842 and 1844, ed. F. Darwin. Cambridge: University Press.

- 1859. On the origin of species by means of natural selection. London: John Murray.

- 1861. On the two forms, or dimorphic condition, in the species of Primula, and on their remarkable sexual relations. Journal of the Proceedings of the Linnean Society of London (Botany) VI: 77-96.

—. 1862. Carta de Darwin a Gray (23/07/1862). En The Correspondence of Charles Darwin - Vol. 10: 1862, eds. Frederick Burkhardt, Joy Harvey, Duncan M. Porter y Jonathan R. Thopman. Cambridge: Cambridge University Press, 1997.

—. 1871. The descent of man. London: John Murray.

- 1872 . The origin of species ( $6^{\text {th }}$ ed.). London: John Murray.

- 1876. The effects of cross and self fertilisation in the vegetable kingdom. London: John Murray.

—. 1877a. The different forms of flowers on plants of the same species. London: John Murray.

— $1877 \mathrm{~b}$. The various contrivances by which orchids are fertilised by insects (2nd edition). London: John Murray.

- 1958. The autobiography of Charles Darwin 1809-1882. With the original omissions restored. Edited and with appendix and notes by his grand-danghter Nora Barlow. London: Collins.

Diez, José A. 2002. A Program for the Individuation of Scientific Concepts. Synthese 130: 13-48.

${ }^{9}$ Incluso si hubiésemos llegado a la conclusión de que TSND y DITN son empíricamente inconmensurables en el sentido señalado, esto no implicaría que no podría haber formas empíricas de compararlas. Pueden encontrarse otros sentidos más débiles en los que ambas teorías 'hablan de lo mismo' aunque no apelen a las mismas teorías para conceptualizar los fenómenos que quieren explicar (Díez 2012; Falguera 2012). Por supuesto esta cuestión es extremadamente controvertida. 
Díez, José A. 2012. Inconmensurabilidad, comparabilidad empírica y escenas observacionales. En Lorenzano y Nudler (2012), 67-118.

Falguera, José Luis. 2012. Comparación epistémica de teorías inconmensurables, sin fundamentismo. En Lorenzano y Nudler (2012), 119-170.

Feyerabend, Paul. 1962. Explanation, Reduction and Empiricism. En Scientific Explanation, Space, and Time, (Minnesota Studies in the Philosophy of Science), eds. Herbert Feigl y Grover Maxwell. Minneapolis: University of Minneapolis Press.

Ghiselin, Michael T. 1969. The triumph of the Darwinian method. Berkeley / London: University of California Press.

Ginnobili, Santiago. 2009a. Adaptación y función. Ludus Vitalis XVII (31): 3-24.

- 2009b. El poder unificador de la teoría de la selección natural. En 150 años después... La vigencia de la teoría evolucionista de Charles Darwin, eds. M. C. Barboza, J. D. Avila, C. Píccoli y J. Cornaglia Fernández. Rosario: Universidad Nacional de Rosario.

- 2010a. La teoría de la selección natural darwiniana. Theoria 25 (1): 37-58

- 2010b. La teoría de la selección natural darwiniana y la genética de poblaciones. Endoxa (24): 169184.

- 2011a. El estatus fenomenológico de la teoría de la selección natural. Ideas y Valores (145): 69-86.

—. 2011b. Función como concepto teórico. Scientiae Studia 9 (4): 847-880.

—. 2011c. Selección artificial, selección sexual, selección natural. Metatheoria 2 (1): 61-78.

- 2012. Reconstrucción estructuralista de la teoría de la selección natural. Agora. Papeles de filosofía 31 (2): 143-169

Gould, Stephen Jay. 1980. The Panda's Thumb. New York: Norton.

Hanson, Norwood Russell. 1958. Patterns of discovery: an inquiry into the conceptual foundations of science. Cambridge: Cambridge University Press.

Hume, David. [1779] 1980. Dialogues Concerning Natural Religion. Indianapolis, Cambridge: Hackett Publishing Company.

Kant, Immanuel. [1781] 1998. Crítica de la razón pura. Buenos Aires: Alfaguara.

Kirby, William. 1836. On the Power, Wisdom and Goodness of God, as Manifested in the Creation of Animals, and in their History, Habits and Instincts. Philadelphia: Carey, Lea \& Blanchard.

Kuhn, Thomas S. 1962. The structure of scientific revolutions. Chicago: University of Chicago Press.

Lennox, James G. 1993. Darwin was Teleologist. Biology and Pbilosophy 8: 409-421.

Lorenzano, Pablo. 2012. Estructuras y aplicaciones intencionales: Inconmensurabilidad teórica y comparabilidad empírica en la historia de la genética clásica. En Lorenzano y Nudler (2012), 289-350.

Lorenzano, Pablo y Oscar Nudler (eds.). 2012. El camino desde Kubn. La inconmensurabilidad hoy. Madrid: Biblioteca Nueva.

Oberheim, Eric y Paul Hoyningen-Huene. 2013. The Incommensurability of Scientific Theories. En The Stanford Encyclopedia of Philosophy. (Spring 2013 edition), Edward N. Zalta ed.

URL $=<$ http://plato.stanford.edu/archives/spr2013/entries/incommensurability/>.

Ospovat, Dov. 1980. God and natural selection: The Darwinian idea of design. Journal of the History of Biology 13: 169-194.

Paley, William. 1809. Natural Theology. London: J. Faulder.

Roget, Peter Mark. 1834. Animal and Vegetable Physiology Considered with Reference to Natural Theology. London: William Pickering.

Sneed, Joseph D. 1971. The Logical Structure of Mathematical Physics. Dordrecht-Holland: Reidel.

Santiago Ginnobili se licenció en la carrera de Filosofía en la Universidad de Buenos Aires orientándose en temas de Filosofía de la Ciencia, especialmente en temas de filosofía de la biología. Luego realizó su doctorado en Filosofía en la misma Universidad. En su tesis doctoral trabajó la teoría de la selección natural desde el enfoque del estructuralismo metateórico. Tiene diversas publicaciones, generalmente sobre temas de filosofía de la biología, en diversas revistas (entre ellas, una reconstrucción de la teoría de la selección natural en un número pasado de esta misma revista) así como varios capítulos de libros. Desde hace unos años es investigador de CONICET, y realiza sus actividades docentes principalmente en la Universidad Nacional de Quilmes y en la Universidad de Buenos Aires.

Direccrón: Instituto de Estudios Sobre la Ciencia y la Tecnología, Universidad Nacional de Quilmes, Roque Sáenz Peña 352, Bernal, Buenos Aires, B1876BXD, Argentina. E-mail: santi75@gmail.com 